\title{
Supporting Information \\ Computer-assisted analysis of microplastics in environmental samples based on $\mu$ FTIR imaging in combination with machine learning
}

by

Benedikt Hufnagl, ${ }^{a, b, *}$ Michael Stibi ${ }^{b}$ Heghnar Martirosyan, ${ }^{c}$ Ursula Wilczek, ${ }^{c}$

Julia N. Möller, ${ }^{c}$ Martin G. J. Löder, ${ }^{c}$ Christian Laforsch ${ }^{c}$ and Hans Lohninger ${ }^{a}$

a Institute of Chemical Technologies and Analytics; Vienna University of Technology; A 1060 Vienna, Austria
benedikt.hufnagl@tuwien.ac.at; https://orcid.org/0000-0002-1470-5787
${ }^{b}$ Purency GmbH, Walfischgasse 8/34, A 1010 Vienna, Austria
${ }^{c}$ Department of Animal Ecology I and BayCEER; University of Bayreuth; D 95440 Bayreuth, Germany; D 95440 Bayreuth, Germany

\section{Enzymatic preparation scheme}

The preparation scheme described by Löder et al. ${ }^{1}$ starts with splitting the sample in two fractions using a $500 \mu \mathrm{m}$ sieve. The larger fraction is sorted manually using a stereo microscope followed by a spectroscopic identification by means of attenuated total reflectance FTIR (ATR-FTIR). The smaller fraction below $500 \mu \mathrm{m}$ is then digested using different enzymes and hydrogen peroxide. A final density separation by means of a zinc chloride solution is applied to remove residual mineral particles. The sample is then filtered through an aluminum oxide filter (Anodisc $0.2 \mu \mathrm{m}$ pore size, $10 \mathrm{~mm}$ diameter) which is the sample carrier for the spectroscopic analysis.

According to Löder et al. ${ }^{1}$ the protocol may be applied to a wide range of different environmental matrices and requires about 16 days while the actual handling time per sample is only about 4 hours. The protocol therefore allows many samples to be processed in parallel and can be optimized for certain matrices as applying all steps will not be necessary in general.

Table S1: Multi-class performance measures. Definitions and scripts for computing the given performance measures can be found in Ballabio et al. ${ }^{2}$.

\begin{tabular}{ll}
\hline Performance measure & Value \\
\hline accuracy & 0.9766 \\
Kappa & 0.9690 \\
ner & 0.9710 \\
Pr & 0.9761 \\
GSn & 0.9708 \\
GPr & 0.9760 \\
GmM & 0.9846 \\
V & 0.9736 \\
VM & 0.9735 \\
F & 0.9736 \\
FM & 0.9734 \\
JM & 0.9695 \\
sInd & 0.9792 \\
EMCC & 0.9690 \\
AUNU & 0.9847 \\
AUNP & 0.9831 \\
AU1U & 0.9986 \\
\hline
\end{tabular}



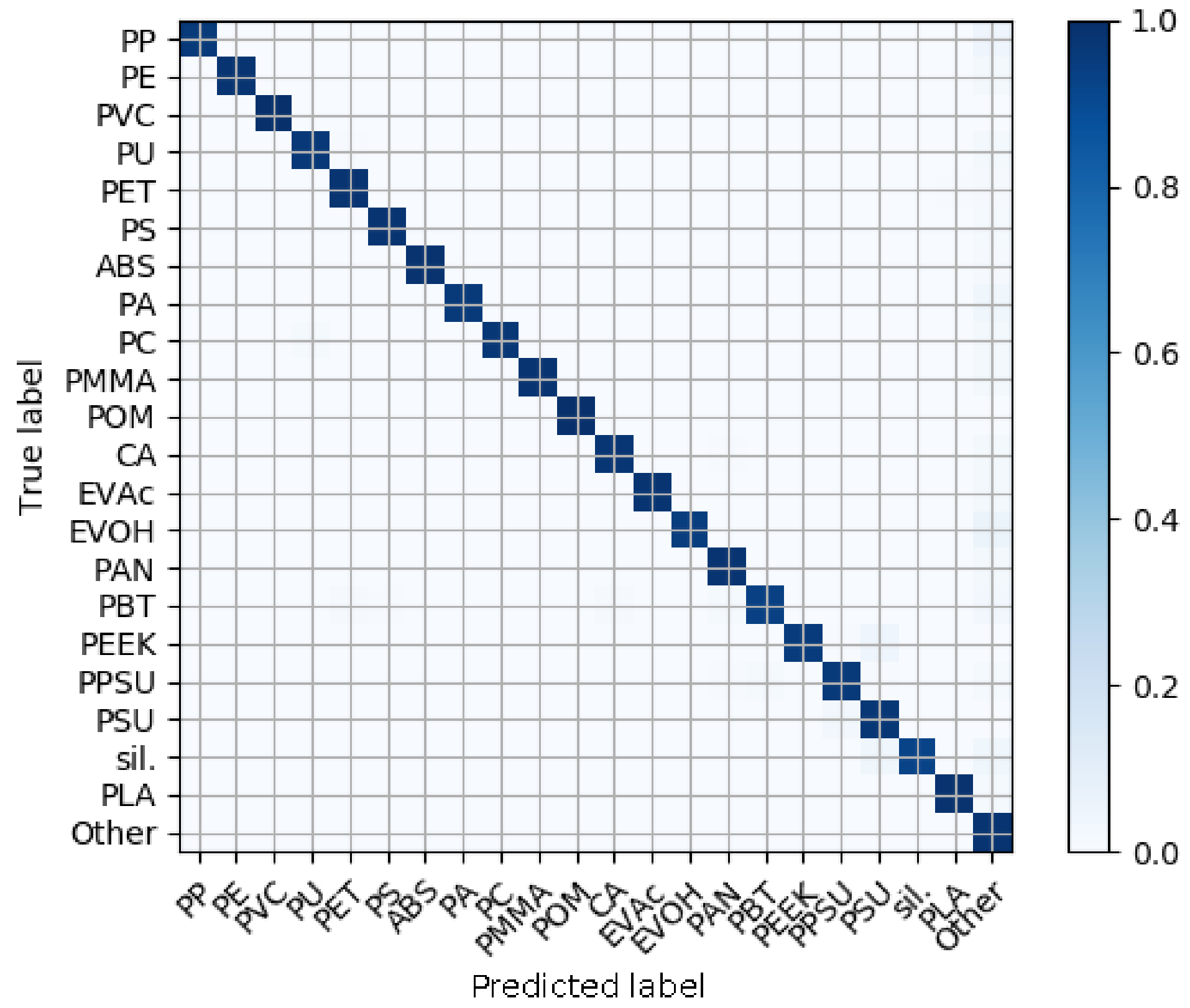

Figure S1: Normalized confusion matrix of the Monte Carlo cross validation. 
Table S2: Original confusion matrix without normalization applied.

\begin{tabular}{|c|c|c|c|c|c|c|c|c|c|c|c|c|c|c|c|c|c|c|c|c|c|c|}
\hline & $\mathrm{PP}$ & $\mathrm{PE}$ & PVC & $\mathrm{PU}$ & PET & PS & ABS & $\mathrm{PA}$ & $\mathrm{PC}$ & PMMA & $\mathrm{CA}$ & EVAc & $\mathrm{EVOH}$ & PAN & PBT & PEEK & $\mathrm{POM}$ & PPSU & PSU & silicone & PLA & Other \\
\hline & 1206 & 0 & 0 & 0 & 0 & 0 & 0 & 0 & 0 & 0 & 0 & 0 & 0 & 0 & 0 & 2 & 0 & 0 & 0 & 0 & 0 & 52 \\
\hline & $\mathrm{PE}$ & 1272 & 0 & 0 & 0 & 0 & 0 & 0 & 0 & 0 & 0 & 0 & 0 & 0 & 0 & 0 & 0 & 0 & 0 & 0 & 0 & 27 \\
\hline & PVC 0 & 0 & 480 & 0 & 0 & 0 & 0 & 0 & 0 & 0 & 0 & 0 & 0 & 0 & 0 & 0 & 0 & 0 & 0 & 0 & 0 & 0 \\
\hline & $\mathrm{PU}$ & 0 & 0 & 619 & 3 & 0 & 0 & 1 & 0 & 2 & 1 & 0 & 0 & 0 & 0 & 0 & 0 & 0 & 0 & 0 & 0 & 14 \\
\hline & PET 0 & 0 & 1 & 0 & 1002 & 0 & 0 & 0 & 0 & 0 & 0 & 0 & 0 & 0 & 0 & 2 & 0 & 0 & 0 & 0 & 4 & 11 \\
\hline & PS 0 & 0 & 0 & 0 & 0 & 707 & 0 & 1 & 0 & 0 & 0 & 0 & 0 & 0 & 0 & 2 & 0 & 0 & 0 & 0 & 0 & 10 \\
\hline & ABS & 0 & 1 & 0 & 0 & 1 & 355 & 0 & 0 & 0 & 0 & 0 & 0 & 0 & 0 & 0 & 0 & 0 & 0 & 0 & 0 & 3 \\
\hline & PA & 1 & 0 & 0 & 0 & 0 & 0 & 1015 & 3 & 0 & 0 & 0 & 0 & 0 & 0 & 0 & 0 & 0 & 0 & 0 & 0 & 41 \\
\hline & $\mathrm{PC}$ & 0 & 0 & 4 & 0 & 0 & 0 & 0 & 330 & 0 & 0 & 0 & 0 & 0 & 0 & 0 & 0 & 0 & 0 & 0 & 0 & 6 \\
\hline & PMMA0 & 0 & 0 & 0 & 0 & 0 & 0 & 0 & 0 & 963 & 0 & 0 & 0 & 0 & 0 & 0 & 0 & 0 & 0 & 0 & 0 & 17 \\
\hline & $\mathrm{CA}$ & 0 & 0 & 0 & 0 & 0 & 0 & 0 & 0 & 0 & 300 & 0 & 0 & 0 & 0 & 0 & 0 & 0 & 0 & 0 & 0 & 0 \\
\hline & EVAc 0 & 0 & 0 & 0 & 0 & 1 & 0 & 0 & 0 & 0 & 0 & 370 & 0 & 0 & 2 & 0 & 0 & 0 & 0 & 0 & 0 & 7 \\
\hline & EVOH 0 & 0 & 0 & 0 & 0 & 0 & 0 & 0 & 0 & 0 & 0 & 0 & 665 & 0 & 0 & 0 & 0 & 0 & 0 & 0 & 0 & 15 \\
\hline & PAN 0 & 0 & 0 & 0 & 0 & 0 & 0 & 0 & 0 & 0 & 0 & 0 & 0 & 284 & 0 & 0 & 0 & 0 & 0 & 0 & 0 & 16 \\
\hline & PBT 0 & 0 & 0 & 0 & 0 & 0 & 0 & 0 & 0 & 0 & 0 & 0 & 0 & 0 & 393 & 0 & 0 & 0 & 0 & 0 & 0 & 7 \\
\hline & PEEK 0 & 0 & 0 & 0 & 3 & 2 & 0 & 0 & 0 & 0 & 0 & 3 & 0 & 0 & 5 & 337 & 0 & 0 & 0 & 0 & 0 & 10 \\
\hline & POM 0 & 0 & 0 & 0 & 0 & 0 & 0 & 0 & 0 & 0 & 0 & 0 & 0 & 0 & 0 & 0 & 286 & 2 & 12 & 0 & 0 & 0 \\
\hline & PPSU 0 & 0 & 0 & 0 & 0 & 0 & 0 & 0 & 0 & 0 & 0 & 0 & 0 & 0 & 2 & 4 & 0 & 328 & 2 & 0 & 0 & 4 \\
\hline & PSU 0 & 0 & 0 & 0 & 0 & 0 & 0 & 0 & 0 & 0 & 0 & 0 & 0 & 0 & 0 & 0 & 0 & 8 & 291 & 0 & 0 & 1 \\
\hline 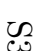 & silicone 0 & 0 & 0 & 0 & 0 & 0 & 0 & 0 & 0 & 0 & 0 & 0 & 0 & 0 & 0 & 0 & 1 & 0 & 10 & 259 & 0 & 10 \\
\hline & PLA 0 & 0 & 0 & 0 & 0 & 0 & 0 & 0 & 0 & 0 & 0 & 0 & 0 & 0 & 0 & 0 & 0 & 0 & 0 & 0 & 730 & 10 \\
\hline & Other 35 & 33 & 8 & 15 & 19 & 11 & 2 & 19 & 7 & 15 & 1 & 1 & 20 & 1 & 3 & 2 & 0 & 5 & 4 & 3 & 10 & 11306 \\
\hline
\end{tabular}




\section{References}

1. Löder, M. G. J.; Imhof, H. K.; Ladehoff, M.; Löschel, L. A.; Lorenz, C.; Mintenig, S.; Piehl, S.; Primpke, S.; Schrank, I.; Laforsch, C.; Gerdts, G. Enzymatic purification of microplastics in environmental samples. Environmental science 85 technology $\mathbf{2 0 1 7}, 51,14283-14292$.

2. Ballabio, D.; Grisoni, F.; Todeschini, R. Multivariate comparison of classification performance measures. Chemometrics and Intelligent Laboratory Systems 2018, 174, 33-44.
3. Hufnagl, B.; Steiner, D.; Renner, E.; Löder, M. G. J.; Laforsch, C.; Lohninger, H. A methodology for the fast identification and monitoring of microplastics in environmental samples using random decision forest classifiers. Analytical Methods 2019, 11, 2277-2285.

4. Primpke, S.; Wirth, M.; Lorenz, C.; Gerdts, G. Reference database design for the automated analysis of microplastic samples based on Fourier transform infrared (FTIR) spectroscopy. Analytical and bioanalytical chemistry 2018, 410, 5131-5141. 


\begin{tabular}{|c|c|c|c|c|c|c|}
\hline PP & PE & PVC & PU & PET & PS & ABS \\
\hline PA & PC & PMMA & POM & CA & EVAC & EVOH \\
\hline PAN & PBT & PEEK & PPSU & PSU & silicone & PLA \\
\hline
\end{tabular}

Figure S2: Associated class colors for the respective 21 polymer types.

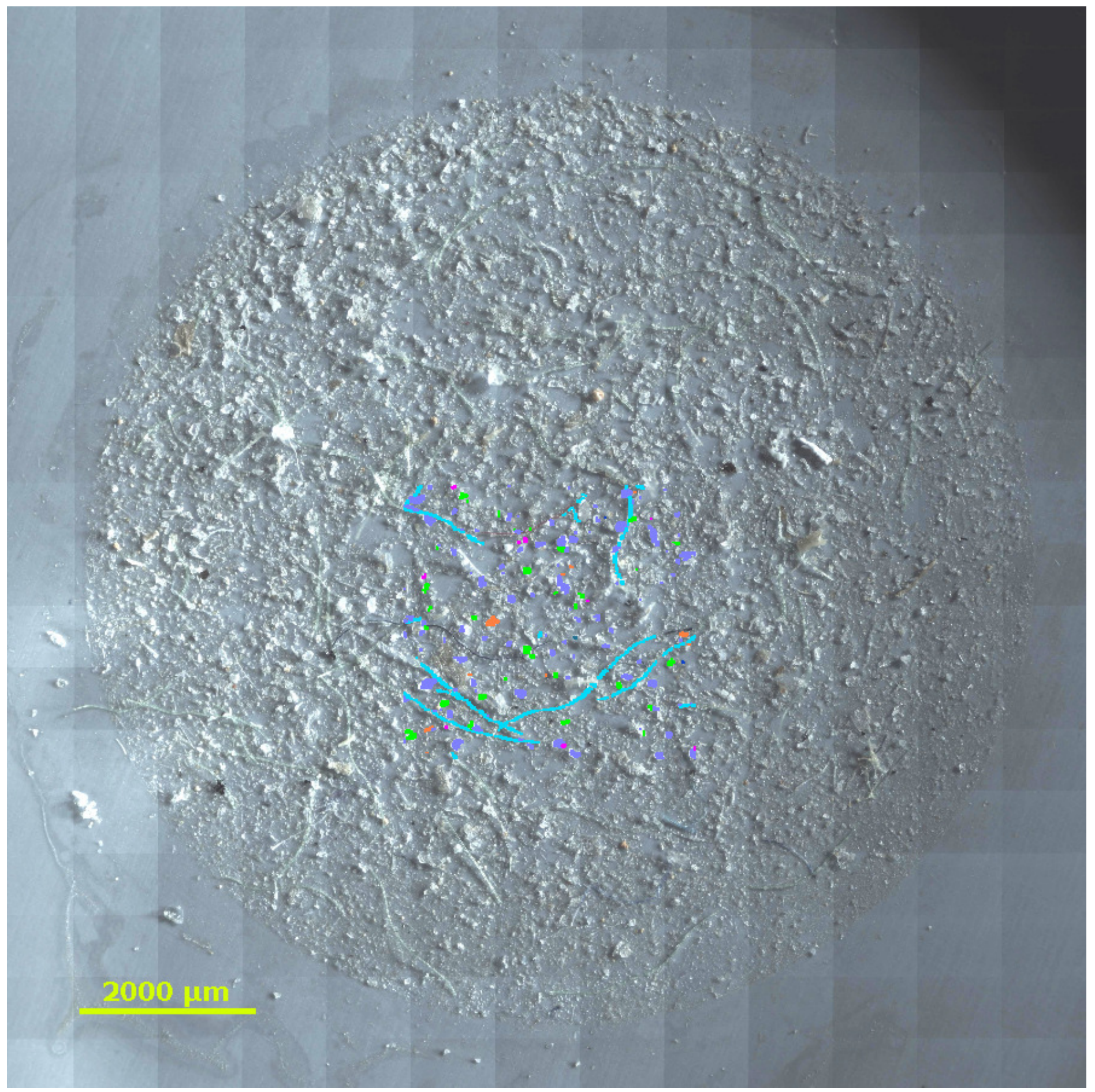

Figure S3: Plankton sample adapted with permission under a Creative Commons Attribution 3.0 Unported License from Hufnagl et al. ${ }^{3}$, Copyright 2019 The Royal Society of Chemistry, original microscope image superimposed with new classification result, DOI: 10.1039/C9AY00252A The original hypercube covered the entire sample area but was trimmed. See figure S2 for the corresponding class colors. 


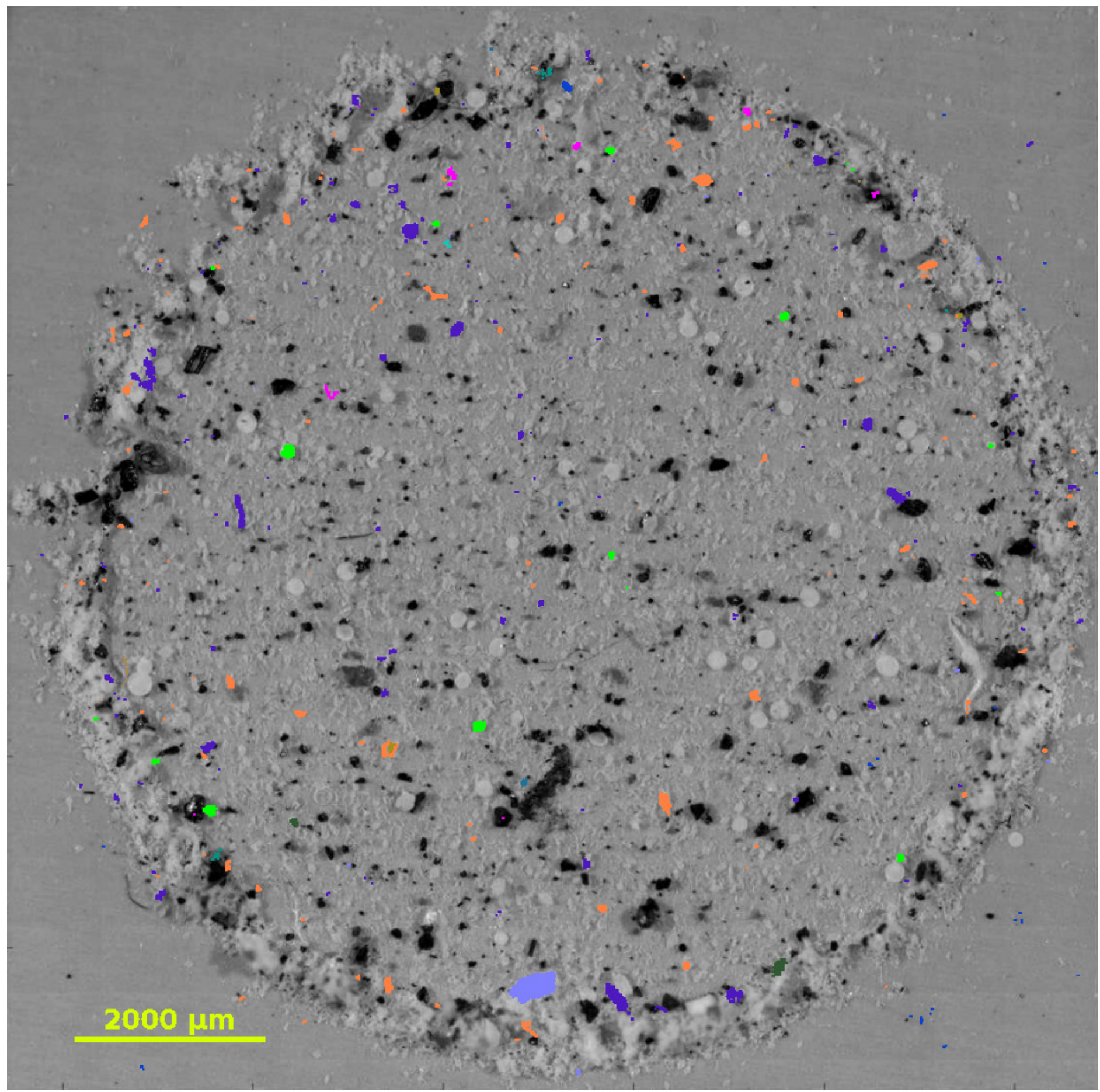

Figure S4: Reference sample 'RefEnv1' adapted with permission under a Creative Commons Attribution 4.0 International License from Primpke et al. ${ }^{4}$, Copyright 2018 Springer Nature, original microscope image superimposed with new classification result, DOI: s00216-018-1156-x. See figure S2 for the corresponding class colors. 


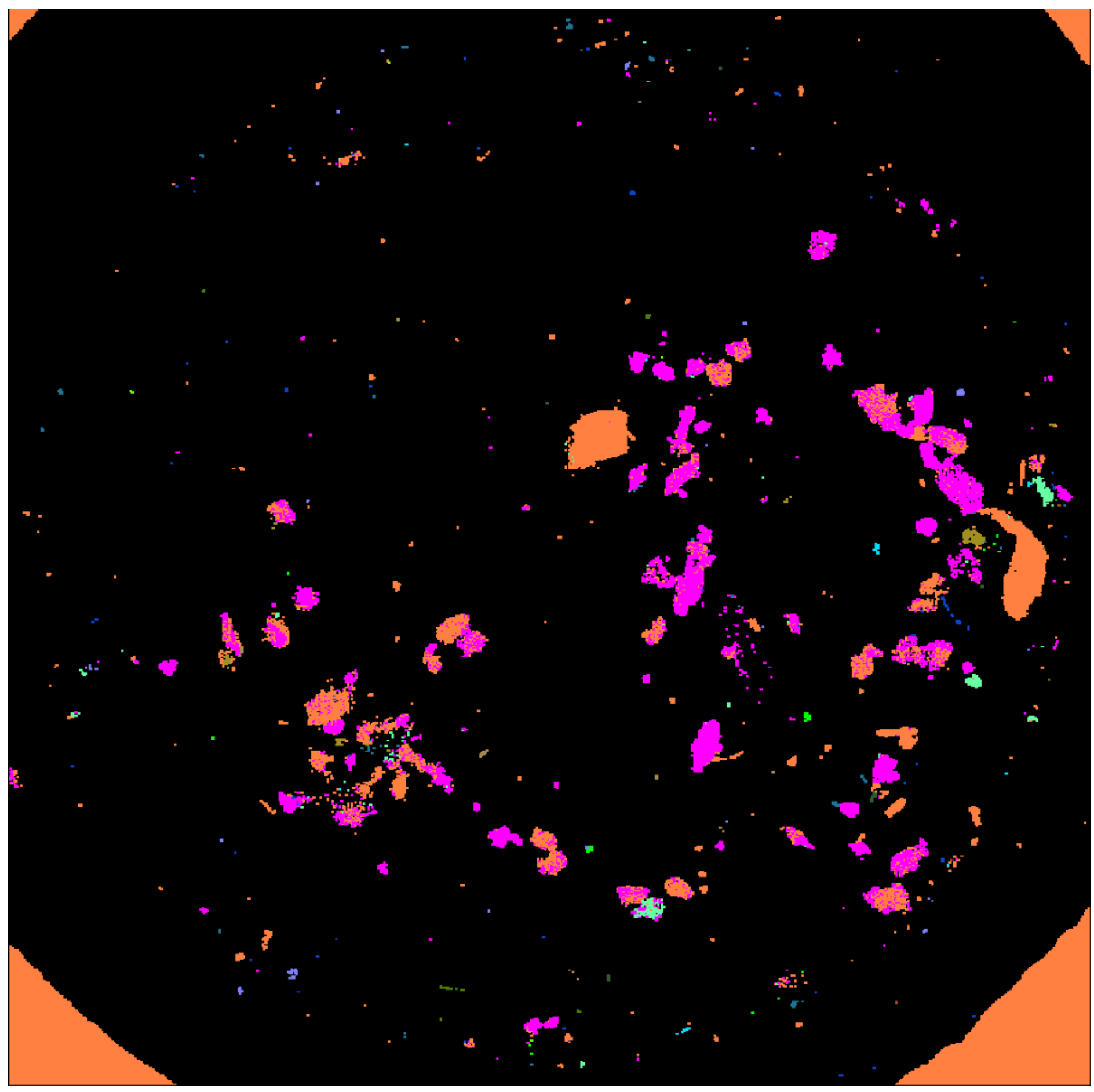

Figure S5: Reference sample 'RefEnv2' adapted with permission under a Creative Commons Attribution 4.0 International License from Primpke et al. ${ }^{4}$, Copyright 2018 Springer Nature, derivative work showing the classification result when the RDF is applied to the dataset, DOI: s00216-018-1156-x. See figure S2 for the corresponding class colors. 




Figure S6: Waste water treatment plant outlet sample. See figure S2 for the corresponding class colors. 


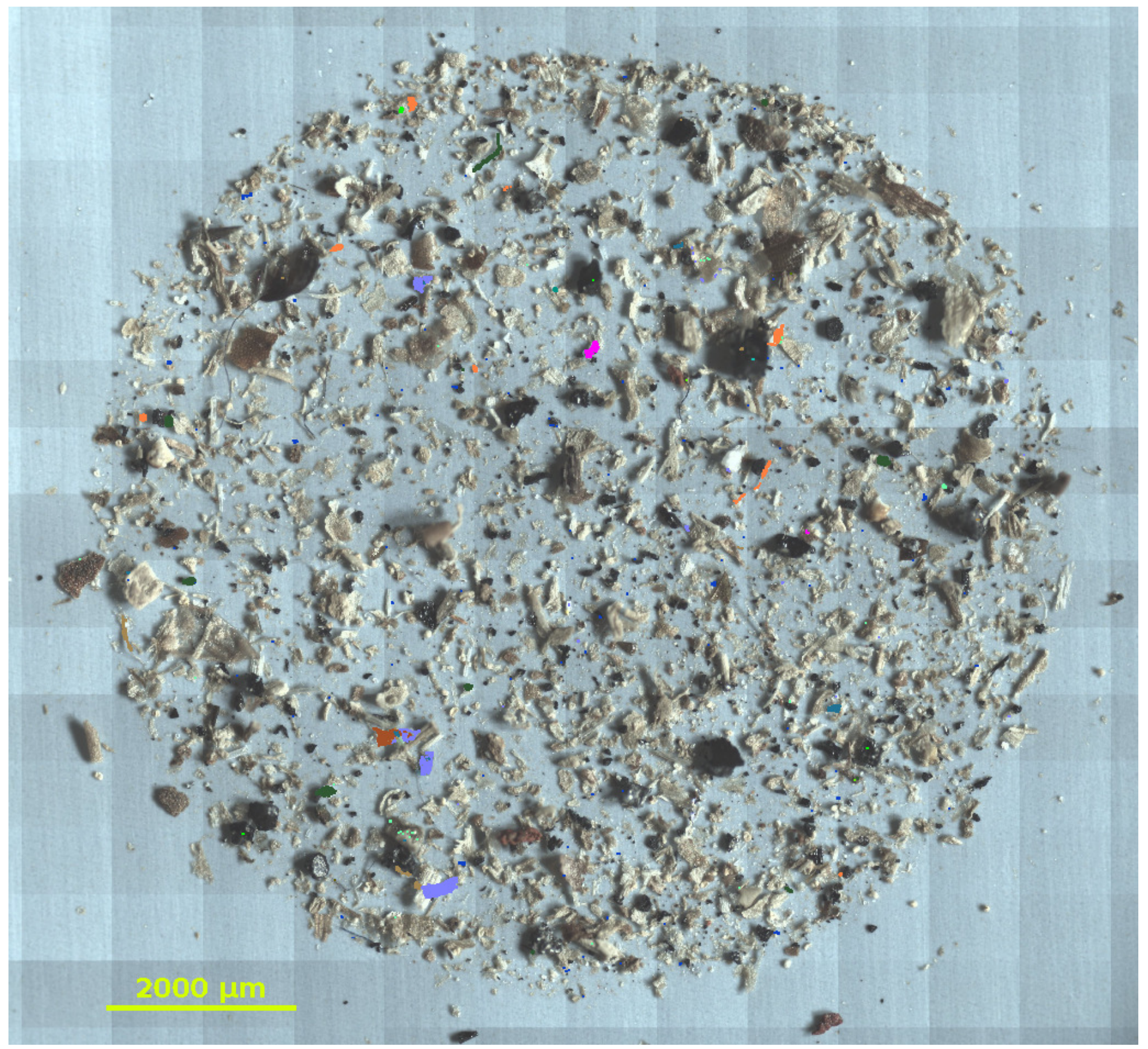

Figure S7: Deep sediment sample. See figure S2 for the corresponding class colors. 


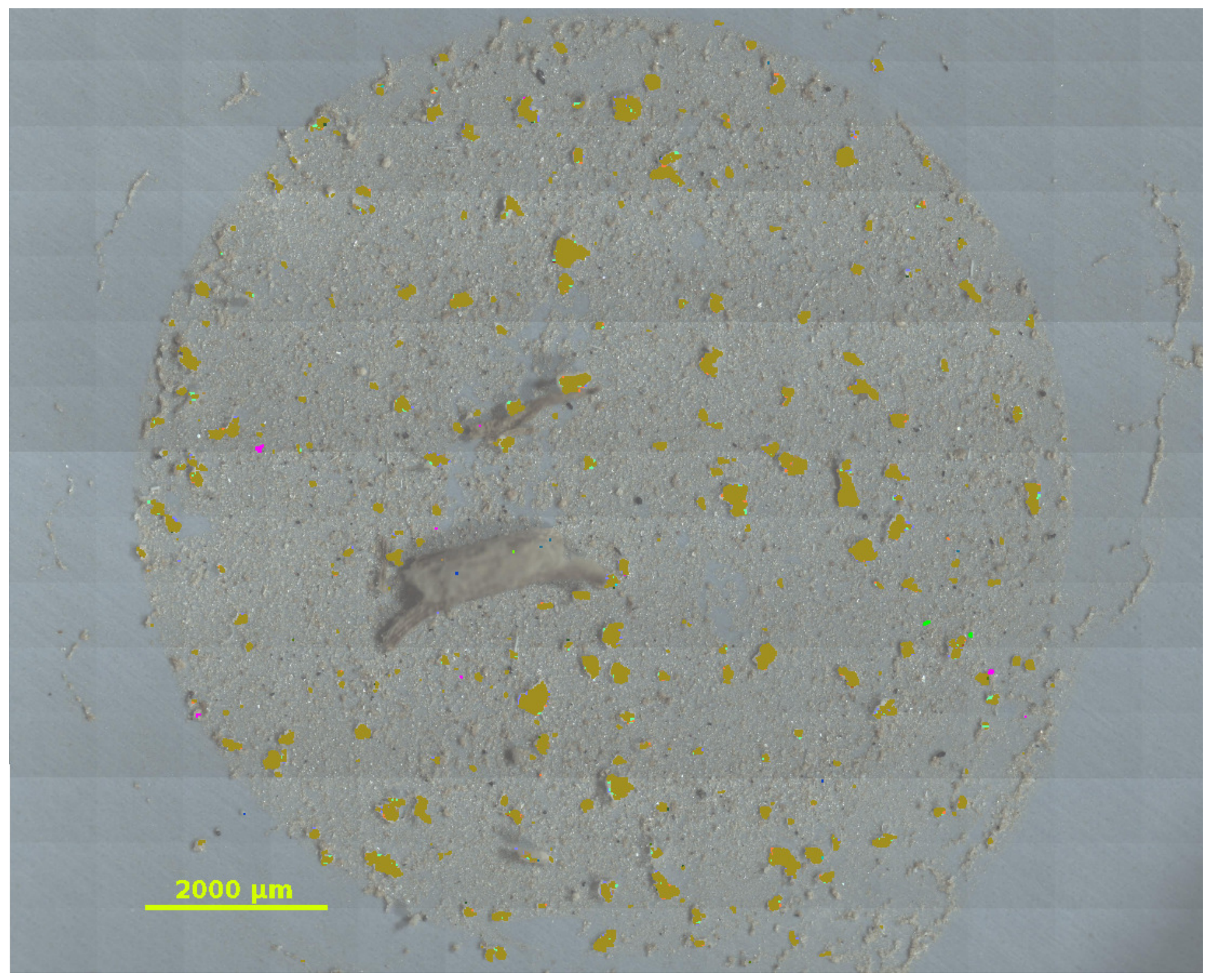

Figure S8: Soil sample. See figure S2 for the corresponding class colors. 


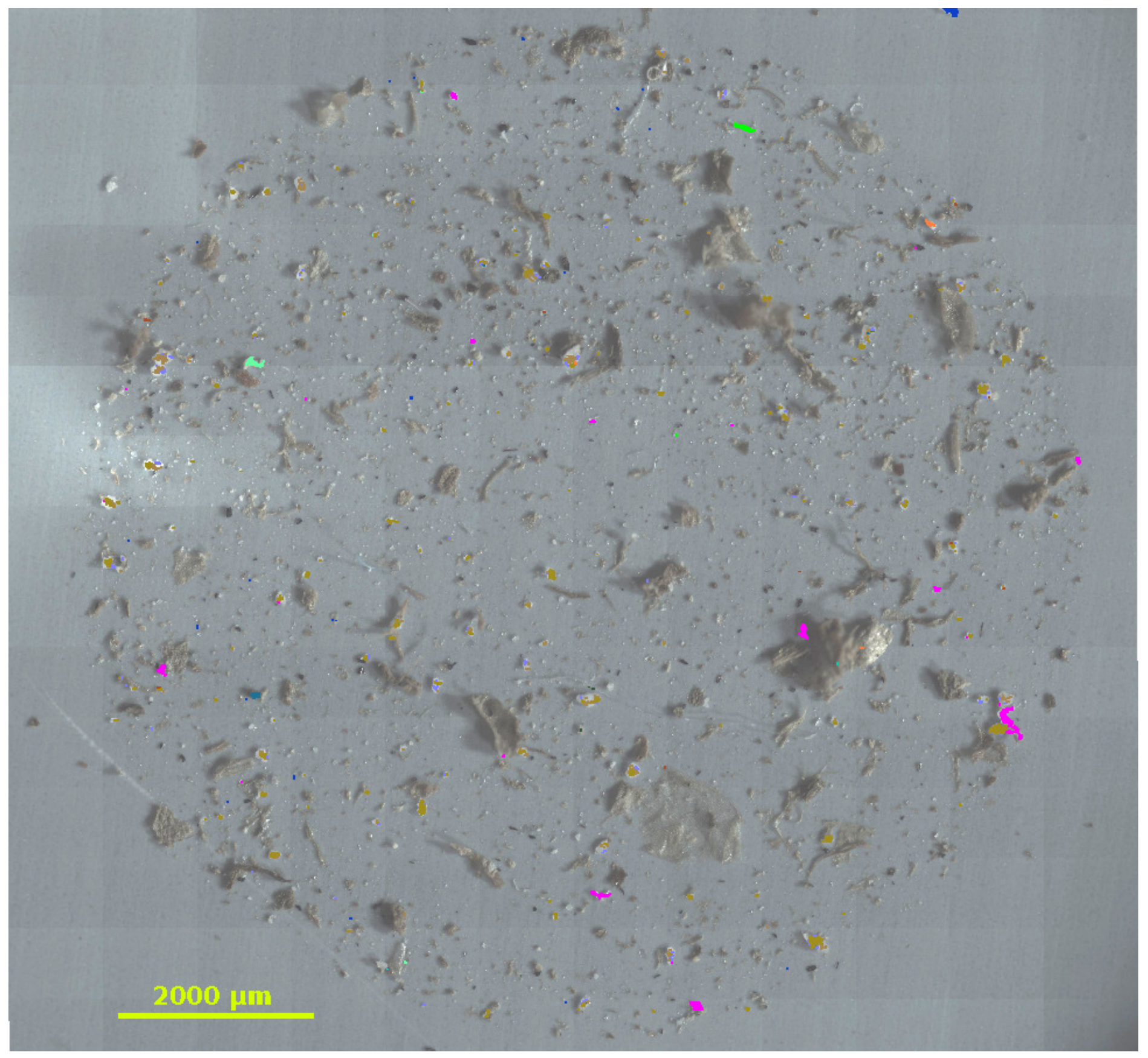

Figure S9: Compost sample. See figure S2 for the corresponding class colors. 


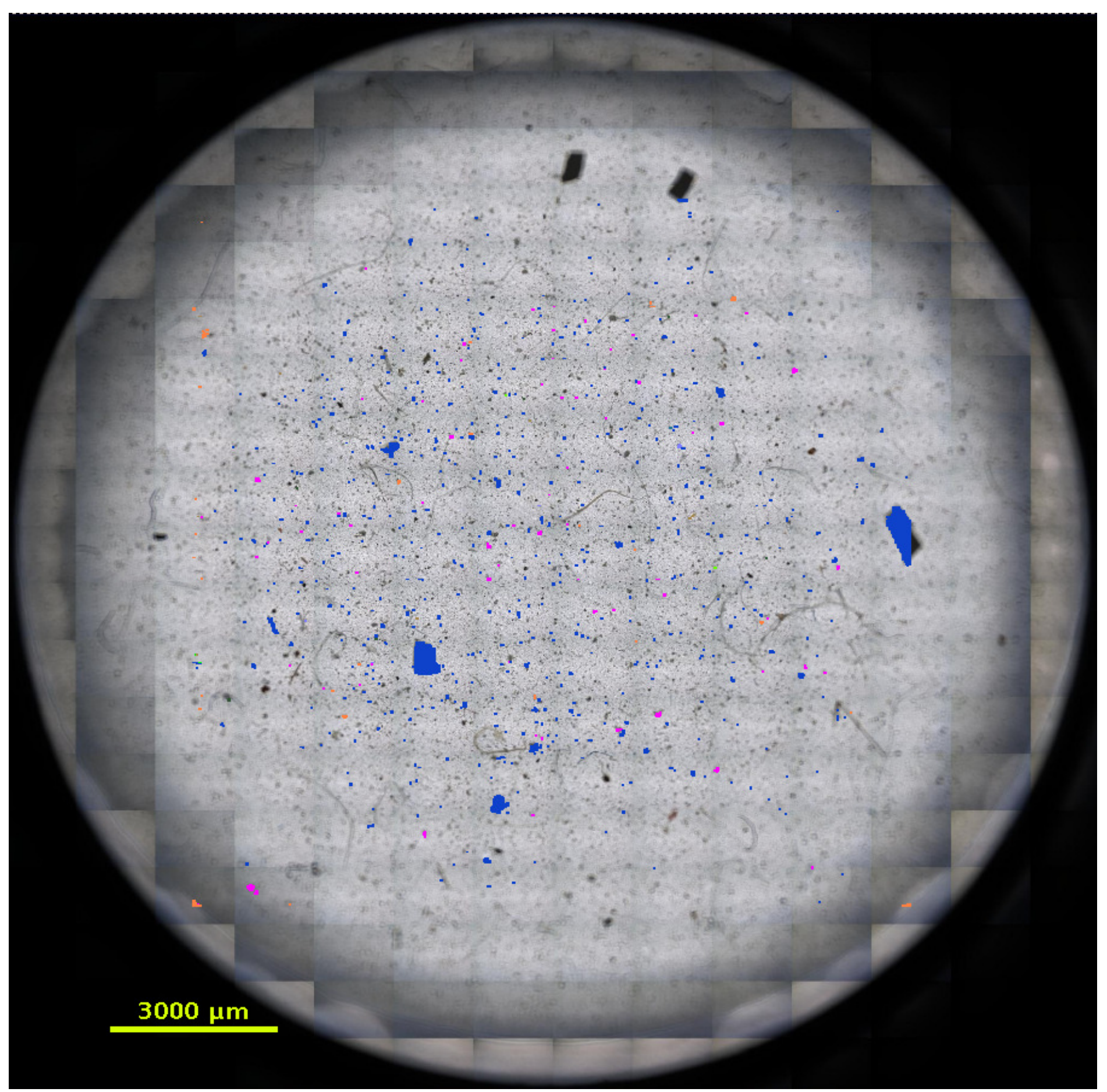

Figure S10: Sea salt sample measured with Bruker LUMOS II. See figure S2 for the corresponding class colors. 


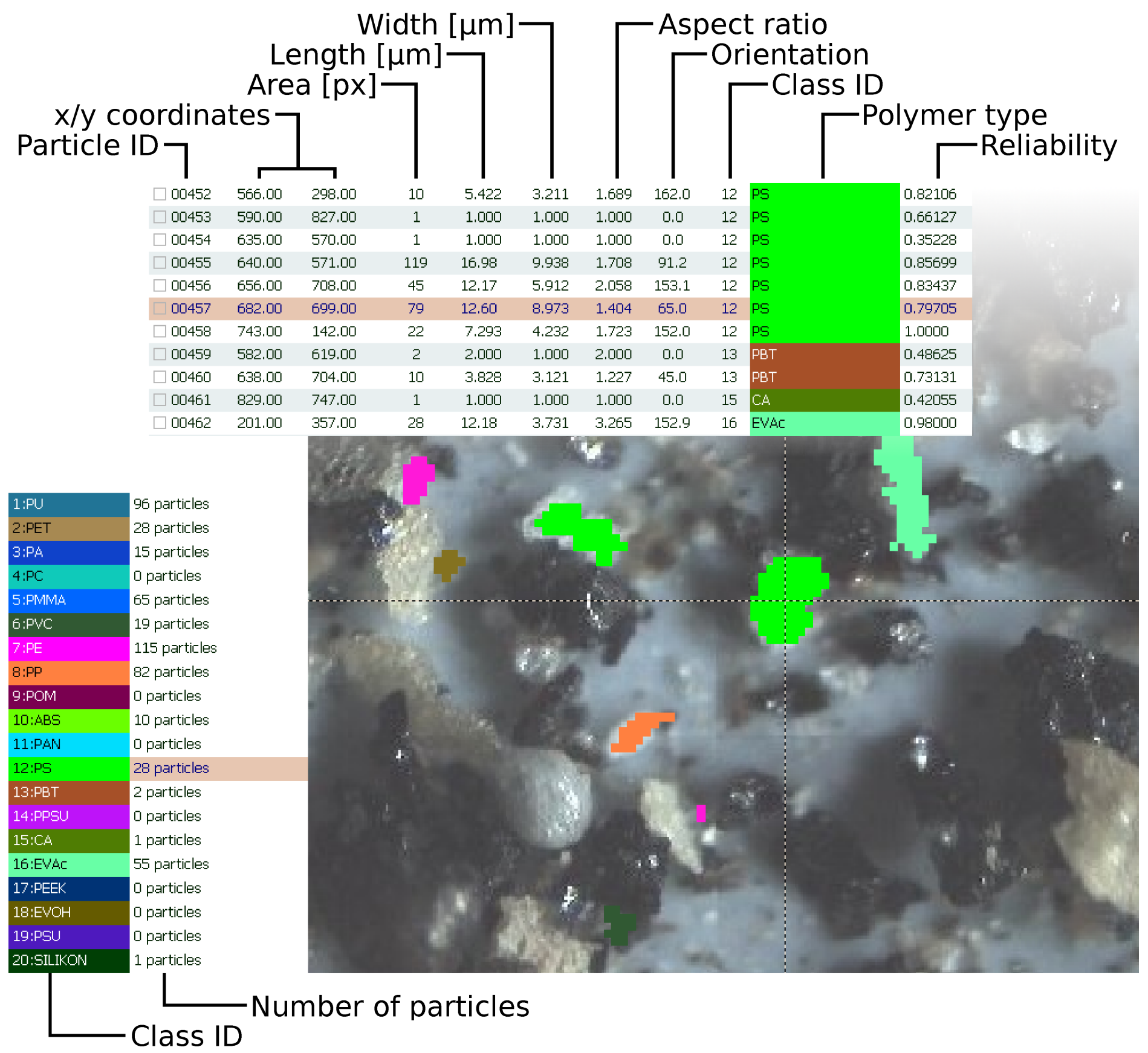

Figure S11: Selected elements of the graphical user interface. 


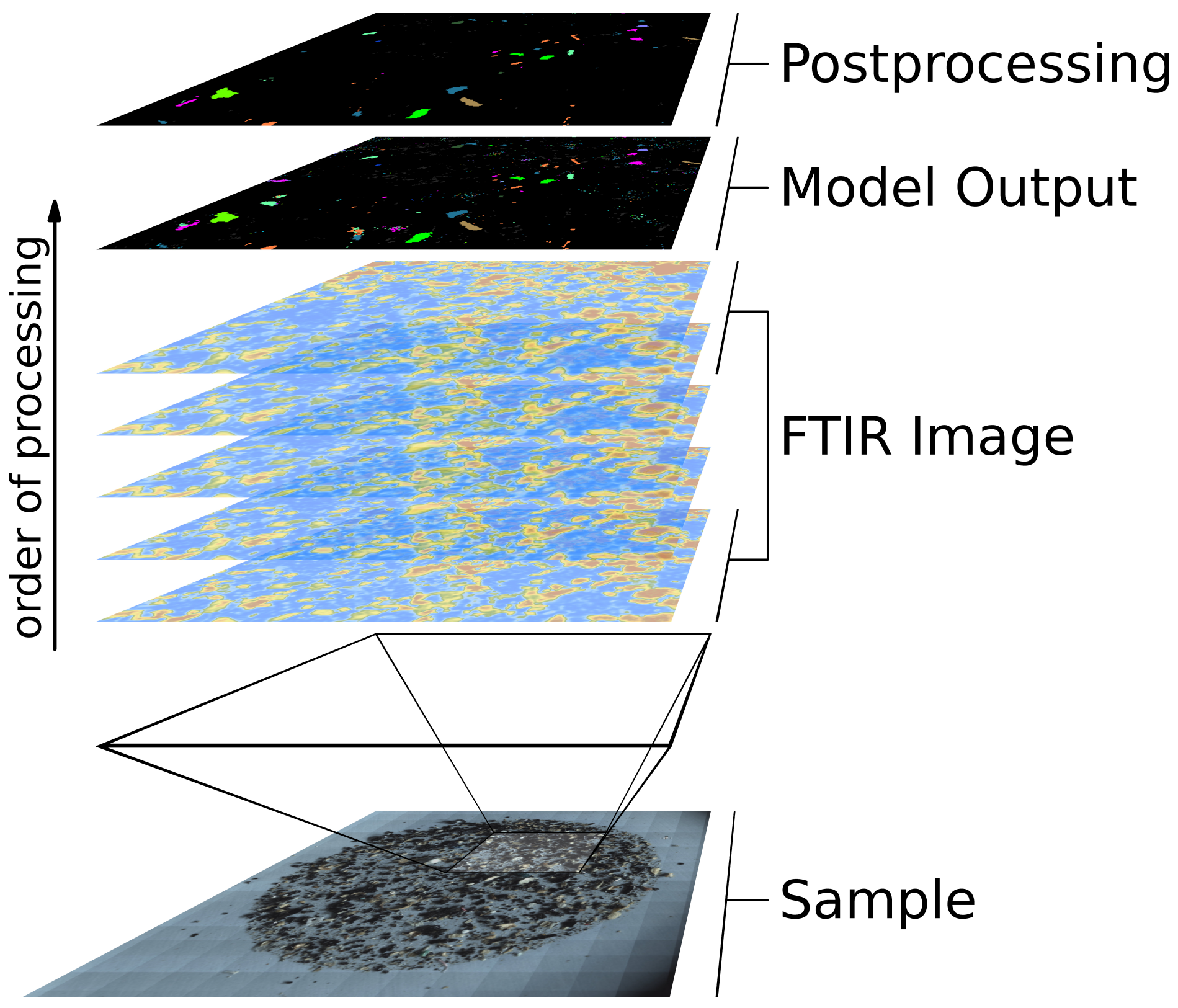

Figure S12: Data acquisition and processing of the Bayreuth Microplastics Finder approach. In this illustration the sample surface is mapped using an FPA-based FTIR imaging spectrometer which produces a high-resolution chemical image. Using a statistical model different microplastics are detected to form an initial model output. In a subsequent step this result is postprocessed and forwarded to the particle characterisation tool which then allows size distributions to be estimated. 